\title{
RESEARCH
}

Open Access

\section{Clinical outcomes of empirical high-dose meropenem in critically ill patients with sepsis and septic shock: a randomized controlled trial}

Tospon Lertwattanachai ${ }^{1}$, Preecha Montakantikul ${ }^{1}$, Viratch Tangsujaritvijit ${ }^{2,3}$, Pitsucha Sanguanwit ${ }^{4}$, Jetjamnong Sueajai ${ }^{5}$, Saranya Auparakkitanon ${ }^{5}$ and Pitchaya Dilokpattanamongkol ${ }^{1 *}$

\begin{abstract}
Background: Appropriate antimicrobial dosing is challenging because of changes in pharmacokinetics (PK) parameters and an increase in multidrug-resistant (MDR) organisms in critically ill patients. This study aimed to evaluate the effects of an empirical therapy of high-dose versus standard-dose meropenem in sepsis and septic shock patients.

Methods: We performed a prospective randomized open-label study to compare the changes of modified sequential organ failure assessment (mSOFA) score and other clinical outcomes of the high-dose meropenem (2-g infusion over $3 \mathrm{~h}$ every $8 \mathrm{~h}$ ) versus the standard-dose meropenem (1-g infusion over $3 \mathrm{~h}$ every $8 \mathrm{~h}$ ) in sepsis and septic shock patients. Patients' characteristics, clinical and microbiological outcomes, 14 and 28-day mortality, vasopressor- and ventilator-free days, intensive care unit (ICU) and hospital-free days, percent of the time of antibiotic concentrations above the minimum inhibitory concentration (\%T>MIC), and safety were assessed.

Results: Seventy-eight patients were enrolled. Median delta mSOFA was comparable between two groups $(-1$ in the high-dose group vs. -1 in the standard-dose group; $P$ value $=0.75)$. There was no difference between the two groups regarding clinical and microbiological cure, 14- and 28-day mortality, vasopressor- and ventilator-free days, and ICU- and hospital-free days. In patients admitted from the emergency department (ED) with a mSOFA score $\geq$ 7, the high-dose group demonstrated significantly better microbiological cure compared with the standard-dose group (75\% (9/12 patients) vs. 20\% (2/10 patients); $P$ value $=0.03)$. Likewise, the high-dose group presented higher microbiological cure rate in patients admitted from ED who had either APACHE II score $>20(83.3 \%(10 / 12)$ vs. $28.6 \%(2 / 7) ; P$ value $=0.045)$ or on mechanical ventilator $(87.5 \%(7 / 8)$ vs. $23.1 \%(3 / 13) ; P$ value $=0.008)$ than the standard-dose group. Adverse events were comparable between the two groups.

(Continued on next page)
\end{abstract}

\footnotetext{
* Correspondence: pitchaya.dil@mahidol.ac.th

'Department of Pharmacy, Faculty of Pharmacy, Mahidol University, Bangkok 10400, Thailand

Full list of author information is available at the end of the article
}

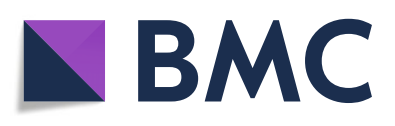

(- The Author(s). 2020 Open Access This article is licensed under a Creative Commons Attribution 4.0 International License, which permits use, sharing, adaptation, distribution and reproduction in any medium or format, as long as you give appropriate credit to the original author(s) and the source, provide a link to the Creative Commons licence, and indicate if changes were made. The images or other third party material in this article are included in the article's Creative Commons licence, unless indicated otherwise in a credit line to the material. If material is not included in the article's Creative Commons licence and your intended use is not permitted by statutory regulation or exceeds the permitted use, you will need to obtain permission directly from the copyright holder. To view a copy of this licence, visit http://creativecommons.org/licenses/by/4.0/ The Creative Commons Public Domain Dedication waiver (http://creativecommons.org/publicdomain/zero/1.0/) applies to the data made available in this article, unless otherwise stated in a credit line to the data. 


\begin{abstract}
(Continued from previous page)
Conclusions: Empirical therapy with the high-dose meropenem presented comparable clinical outcomes to the standard-dose meropenem in sepsis and septic shock patients. Besides, subgroup analysis manifested superior microbiological cure rate in sepsis or septic shock patients admitted from ED.
\end{abstract}

Trial registration: ClinicalTrials.gov, NCT03344627, registered on November 17, 2017

Keywords: Meropenem, High dose, Sepsis, Septic shock, Empirical therapy, Clinical outcome

\section{Background}

Sepsis is described as a serious organ dysfunction which results from response of dysregulated host to infection. This syndrome is an important healthcare problem that affects significant morbidity and mortality [1]. Early identification of sepsis and aggressive management in first hour are cornerstones for improving patient outcomes [2]. Delay in an initial appropriate antimicrobial therapy has been correlated with an increase in mortality [3]. The initial selection of appropriate antimicrobial is defined in terms of broad-spectrum and adequate dosing of selected antimicrobial for suspected pathogens [3]. However, appropriate antimicrobial dosing is challenging because of altered pharmacokinetics (PK) and increased multidrug-resistant (MDR) organisms in critically ill patients [4]. A larger volume of distribution, which is caused by fluid resuscitation and capillary leak syndrome, culminates in subtherapeutic levels of antimicrobial [5]. The fluctuation of renal clearance, such as augmented renal clearance or acute kidney injury, could affect by increasing or decreasing antimicrobials concentrations [6]. In addition, Suwantarat et al. reported a high prevalence of MDR gram-negative bacteria in Thailand, including $28.7 \%$ for carbapenem-resistant Pseudomonas aeruginosa, $45.2 \%$ for extended-spectrum beta-lactamases (ESBL) producers, and $76.3 \%$ for carbapenem-resistant Acinetobacter baumannii [7]. Due to the spread of antimicrobial-resistant organisms, as well as a decline in new antimicrobial developments, the exploration for effective antimicrobial activity based on PK and pharmacodynamic (PD) properties is the solution [4]. Moreover, the standard recommended dose of antibiotic, which was derived from (1) PK data from healthy volunteers or non-intensive care unit (ICU) patients, and (2) the minimum inhibitory concentration (MIC) for non-resistant bacteria, may increase the risk of treatment failure [4]. Roberts et al. performed a PK study of 8 beta-lactam antibiotics in critically ill patients across 68 ICUs in 10 European countries. From a total of 248 patients, $16 \%$ of patients did not achieve $50 \%$ of the time of free antibiotic concentrations above the minimum inhibitory concentration $(\mathrm{f} T>\mathrm{MIC})$ of the pathogen, and these patients were less likely to complete a treatment course and achieve positive clinical outcome with the initially selected beta-lactam [8].
Meropenem, a member of the carbapenem class, is widely used as empirical therapy in the treatment of sepsis and septic shock regarding its broad-spectrum activity and a low toxicity profile. The PD of meropenem is a time-dependent activity, similar to other beta-lactam antibiotics. At least $40 \% \mathrm{fT}>\mathrm{MIC}$ is required for carbapenems to achieve their bactericidal activity [4]. However, to achieve favorable microbiological and clinical outcomes for critically ill patients with severe infections, achievement of $100 \% \mathrm{fT}>\mathrm{MIC}$ is required, and prolonged infusion of meropenem is recommended $[6,9]$. Additionally, the standard recommended dose of meropenem was not sufficient to achieve a higher PK/PD target in sepsis and septic shock patients $[4,10,11]$. Jaruratanasirikul et al. conducted a population PK study of meropenem in critically ill patients. They also indicated that the maximum recommended dose of $2 \mathrm{~g}$ of meropenem every $8 \mathrm{~h}$ may be needed for an early phase of sepsis and septic shock [12].

Currently, there was a lack of study of the high-dose meropenem in sepsis and septic shock patients. We proposed that the given high-dose, prolonged infusion regimen in early phase sepsis and septic shock would help reduce mortality, defined by a reduction in modified sequential organ failure assessment (mSOFA) score. Therefore, we designed a randomized study to compare the changes in mSOFA score and other clinical outcomes of the standard-dose versus the high-dose meropenem in critically ill patients with sepsis and septic shock.

\section{Methods \\ Design and settings}

This study was a prospective, single-center, randomized, open-label study conducted at an academic hospital in Bangkok, Thailand. This trial was registered in ClinicalTrial.gov identifier NCT03344627 and was approved by the committee on human rights related to research, Faculty of Medicine Ramathibodi Hospital, Mahidol University.

\section{Inclusion and exclusion criteria}

The inclusion criteria included (1) patients aged $>18$ years, (2) patients diagnosed as sepsis and septic shock according to sepsis-3 criteria and received meropenem within $1 \mathrm{~h}$ after diagnosis, (3) patients (or relatives) signing the consent form. The exclusion criteria included (1) patients having sepsis with central nervous system infection, infective endocarditis; (2) patients requiring 
surgical condition within $72 \mathrm{~h}$ after randomization; (3) patients receiving extracorporeal membrane oxygenation (ECMO) within 3 days after randomization; (4) patients with active seizure; (5) patients receiving meropenem within 1 week prior to randomization; (6) pregnancy or lactation; (7) patients having known allergy to meropenem; and (8) patients receiving meropenem as empirical therapy less than 3 days.

\section{Patient randomization and treatment protocol}

Patients were randomized using a sealed opaque envelope in a block of four stratified according to their status prior to ICU admission (escalated from other wards or admitted to ICU from emergency department (ED)). They were assigned to receive either standard-dose meropenem (the standard-dose group, $1 \mathrm{~g}$ of meropenem intravenous (IV) infused over $30 \mathrm{~min}$, then $1 \mathrm{~g}$ of meropenem IV infused over $3 \mathrm{~h}$ every $8 \mathrm{~h}$ ) or high-dose meropenem (the high-dose group, $2 \mathrm{~g}$ of meropenem IV infused over $30 \mathrm{~min}$ then $2 \mathrm{~g}$ of meropenem IV infused over $3 \mathrm{~h}$ every $8 \mathrm{~h}$ ). The study was an open-label study in which the dose was assigned according to the randomization. The enrolled patients were treated using a standard supportive treatment in accordance with the assignment of the meropenem group. The investigator had not been involved in other treatments. Creatinine clearance $(\mathrm{ClCr})$ was calculated by the Cockcroft formula. The dosage of meropenem during the study period was adjusted according to $\mathrm{ClCr}$. It should be noted that patients were allowed to receive concomitant antimicrobial for polymicrobial infections. De-escalation should be narrower spectrum antibiotics according to patient-specific microbiological culture. The recommended duration of antibiotic treatment was based on the decision of the ICU physician team.

\section{Data collection and clinical endpoints}

Baseline characteristics such as age, gender, weight, type of admission, Acute Physiology and Chronic Health Evaluation II (APACHE II), mSOFA scores, concomitant antimicrobial therapy, and source of infection were collected at the start of meropenem therapy.

The primary outcome was delta mSOFA scores (differences between mSOFA scores at day 4 after randomization and mSOFA score at day 1 after randomization). Secondary outcomes were 14-day mortality, 28-day mortality, clinical cure, microbiological cure, vasopressor-free days, ventilatorfree days, ICU- and hospital-free days, and safety of both dosing regimen groups. Clinical cure was defined as a complete or partial resolution of fever (temperature > $38.3^{\circ} \mathrm{C}$ ) for more than $24 \mathrm{~h}$ and leucocytosis (white blood cell count $>12 \times 10^{9} / \mathrm{L}$ ). Microbiological cure was defined as eradication of the study entry pathogen at the suspected site(s) of infection within 14 days (collected at day 3, 5, 7, 10, and 14) after giving meropenem. Blood samples for meropenem level were obtained and measured by liquid chromatography-tandem mass spectrometry (LC-MS/MS) to calculate an achievement of the PD target. Superinfection was defined as recently developed infections by the Centers for Disease Control and Prevention (CDC) criteria within 14 days after being given meropenem, or any newly discovered carbapenem-resistant or colistin-resistant gram-negative bacteria. Vasopressor, ventilator, ICU, and hospital-free days were counted as $28-X$ if successfully liberated from vasopressor use, mechanical ventilation, ICU stay or hospital stay $X$ days after the first day of being given meropenem. Safety outcome was recorded daily during the course of antibiotic treatment.

\section{Statistical analysis}

The sample size was calculated based on our preliminary study, the patient who received meropenem and survived from sepsis and septic shock had shown a reduction in delta mSOFA score of 1.850 (standard deviation (SD) of 2.961). To show a reduction in mSOFA score of 2 in the high-dose group, a sample size of 39 patients in each group was deemed necessary with type I error of $5 \%$, power of $80 \%$, and predicted $10 \%$ dropout from the study.

The per-protocol approach was adopted to make inference on the primary outcome. The KolmogorovSmirnov test was selected for the check of the data distribution. Continuous normally distributed data and non-normally distributed data were tested using unpaired $t$ test and Mann-Whitney $U$ test, respectively. Categorical data were tested by Chi-square or Fisher's exact test, as appropriate. Normally distributed data and non-normal distributed data are presented as mean + SD and median with interquartile range. A two-sided $P$ value $<0.05$ was considered statistically significant for all tests. All statistical calculations were performed with the SPSS statistical package version 18.0.

\section{Results}

Five hundred and twenty-seven patients were screened between August 2017 and October 2018, of whom 451 patients failed to meet the inclusion criteria. The remaining 76 patients were enrolled in the study (Fig. 1). Thirty-eight patients were allocated to each of the standard-dose group and the high-dose group. Age, gender, weight, underlying diseases, $\mathrm{ClCr}$, type of admission, the severity of illness, and source of infection were comparable between the two groups. The major source of infection in the standard-dose meropenem group was respiratory $(39.5 \%)$, bloodstream $(31.6 \%)$, multiple sources $(13.2 \%)$, and urinary tract $(5.3 \%)$. The main source of infection in the high-dose meropenem group was respiratory $(42.1 \%)$, followed by urinary tract 


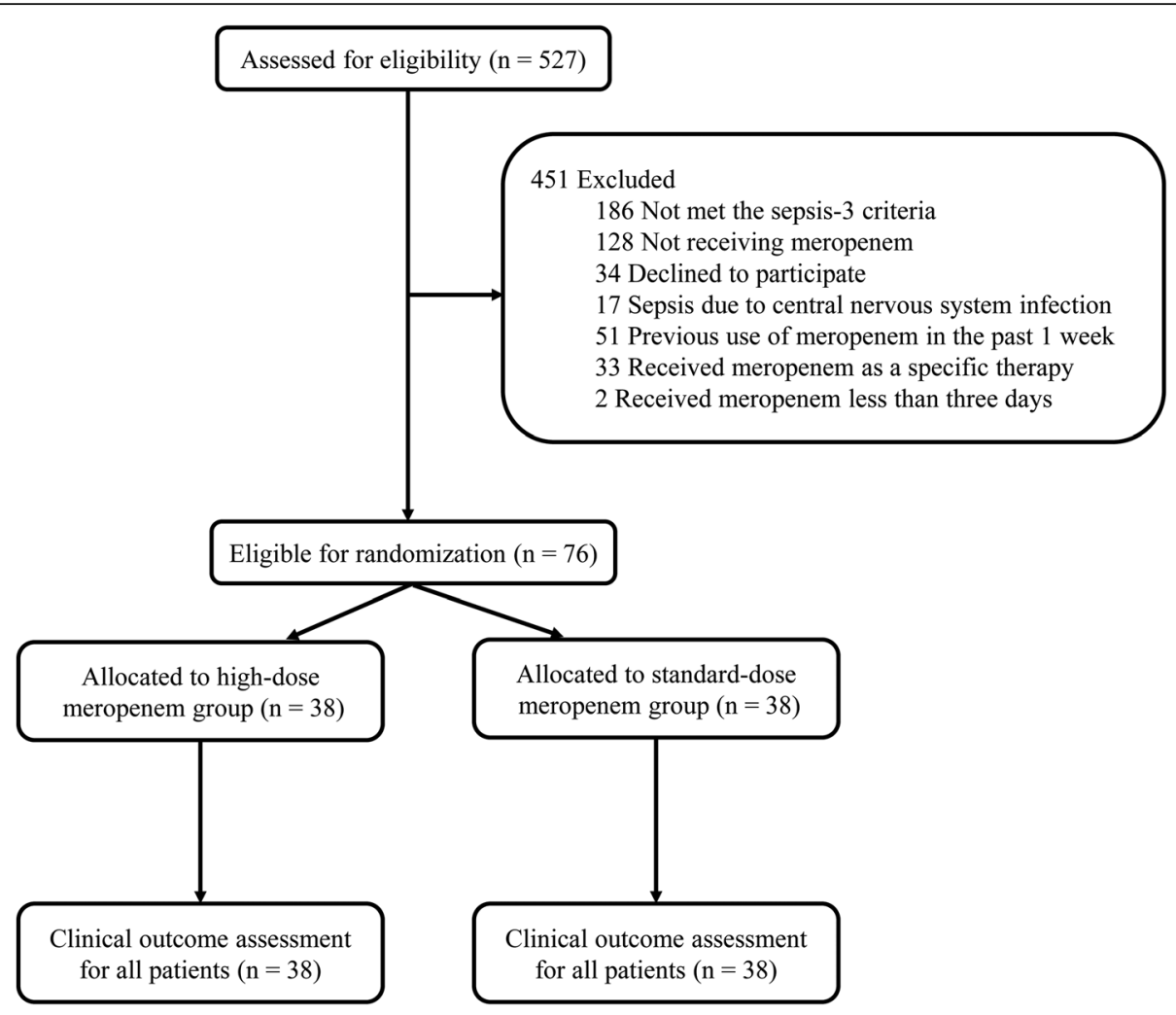

Fig. 1 Flow of patients through the trial

(23.7\%), multiple sources (21.2\%), and bloodstream (18.4\%) as shown in Table 1.

The median duration of meropenem treatment was significantly higher in the standard-dose group (6 (3 to 16) days vs. 5 (3 to 21$)$ days; $P$ value $=0.035$ ). Concomitant antimicrobial therapy was used in $42.1 \%$ in the standard-dose group and $50 \%$ in the high-dose group. The antibacterial drugs comprised of multiple classes, such as polymyxin, glycopeptides, aminoglycosides, and fluoroquinolones. The types of concomitant antibiotics were not significantly different between groups (Table 2). De-escalations from meropenem to narrow-spectrum antibiotics were more than $50 \%$ in both groups.

The microbiological culture was positive in $55.3 \%$ in the standard-dose group and $60.5 \%$ in the high-dose group. There was no significant difference regarding culture positive for polymicrobial, gram-positive bacteria and gram-negative bacteria (Table 1). Similar results for gram-negative bacterial isolates showed no difference, and more than $80 \%$ were susceptible to meropenem (Fig. 2). The most common organisms were Enterobacteriaceae, Pseudomonas aeruginosa, and Acinetobacter baumannii, respectively, in patients with culture-positive gram-negative bacteria (Table 1 ).

Delta mSOFA score was comparable between the high-dose and the standard-dose group ( -1 ( -9 to 4$)$ vs. -1 ( -6 to 3$) ; P$ value $=0.746)$. Delta cardiovascular score was decreased (1-point reduction) in the high-dose group compared to no change (no point reduction) in the standard-dose group, but this was not statistically significant. For the secondary outcome, the clinical cure rate was not different $(86.8 \%$ in the high-dose vs. $86.8 \%$ in the standard-dose group; $P$ value $=1.00)$. Lower $14-$ day mortality and 28-day mortality were observed in the high-dose group (10.5\% vs. $15.8 \%$; $P$ value $=0.736)$ and $(34.2 \%$ vs. $44.7 \%$; $P$ value $=0.482)$, respectively. The microbiological cure had a trend to better cure rate in the high-dose group than the standard-dose group $(55.3 \%$ vs. $44.7 \%$; $P$ value $=0.492)$. Vasopressor-free days, ventilator-free days, ICU-free days, and hospitalfree days were not significantly different between the treatment groups (Table 3). Meropenem levels were obtained from eighteen patients (8 patients in the standard-dose group and 10 patients in the high-dose groups). Regarding the identified MICs, PD target of $40 \%$ of the time above identified MIC was entirely achieved in all patients. However, the PD target of $100 \%$ of the time above the identified MIC was achieved in $66.7 \%$ (2/8 patients) in the standard-dose group and $100 \%$ (5/5 patients) in the high-dose group with no statistically significant difference between groups $(P$ value $=$ 0.375) (Table 4). 
Table 1 Demographics and baseline characteristics

\begin{tabular}{|c|c|c|c|}
\hline Characteristics & Standard-dose meropenem $(n=38)$ & High-dose meropenem $(n=38)$ & $P$ value \\
\hline Age (years), mean (SD) & $67(14.6)$ & $69(19.0)$ & 0.657 \\
\hline Male, $n(\%)$ & $21(55.3)$ & $19(50)$ & 0.819 \\
\hline Weight (kg), mean (SD) & $56.4(12.4)$ & $57.2(14.7)$ & 0.797 \\
\hline \multicolumn{4}{|l|}{ Underlying disease, $n$ (\%) } \\
\hline Diabetes & $13(34.2)$ & $7(18.4)$ & 0.192 \\
\hline Hypertension & $17(44.7)$ & $15(39.5)$ & 0.817 \\
\hline Heart failure & $5(13.2)$ & $3(7.9)$ & 0.711 \\
\hline Chronic kidney disease & $5(13.2)$ & $5(13.2)$ & 1.000 \\
\hline End stage renal disease & $6(15.8)$ & $1(2.6)$ & 0.108 \\
\hline Chronic liver disease & $4(10.5)$ & $3(7.9)$ & 1.000 \\
\hline COPD & $8(21.1)$ & $2(5.3)$ & 0.086 \\
\hline Hematologic malignancy & $7(18.4)$ & $8(21.1)$ & 1.000 \\
\hline Solid malignancy & $8(21.1)$ & $7(18.4)$ & 1.000 \\
\hline Neutropenia & $4(10.5)$ & $7(18.4)$ & 0.516 \\
\hline ARDS & $13(34.2)$ & $10(26.3)$ & 0.628 \\
\hline Cockroft-Gout ClCr (mL/min), median (IQR) & $41.81(7.36-166.8)$ & $30.37(7.04-141.75)$ & 0.377 \\
\hline \multicolumn{4}{|l|}{ Type of admission, $n$ (\%) } \\
\hline ED & $17(44.7)$ & $18(47.4)$ & 1.000 \\
\hline Ward & $21(55.3)$ & $20(52.6)$ & \\
\hline Mechanical ventilator, $n(\%)$ & $29(76.3)$ & $27(71.1)$ & 0.795 \\
\hline APACHE II score, mean (SD) & $18.8(5.7)$ & $20(6.3)$ & 0.395 \\
\hline mSOFA score, median (IQR) & $7(0-13)$ & $8(1-16)$ & 0.088 \\
\hline Septic shock, $n(\%)$ & $26(68.4)$ & $27(71.1)$ & 1.000 \\
\hline \multicolumn{4}{|l|}{ Source of infection } \\
\hline Unknown, $n(\%)$ & $9(23.7)$ & $6(15.8)$ & 0.565 \\
\hline Respiratory, n (\%) & $15(39.5)$ & $16(42.1)$ & 1.000 \\
\hline Urinary tract, $n(\%)$ & $2(5.3)$ & $9(23.7)$ & 0.047 \\
\hline Bloodstream, $n(\%)$ & $12(31.6)$ & $7(18.4)$ & 0.289 \\
\hline Multiple sources, $n(\%)$ & $5(13.2)$ & $8(21.2)$ & 0.540 \\
\hline Culture, $n(\%)$ & $21(55.3)$ & $23(60.5)$ & 0.817 \\
\hline Polymicrobial & $6(28.6)$ & $3(13.0 \%)$ & 0.272 \\
\hline Gram-positive pathogens & $5(23.8)$ & $1(4.3)$ & 0.088 \\
\hline Gram-negative pathogens & $16(76.2)$ & $20(87.0)$ & 0.448 \\
\hline Escherichia coli & 7 (33.3) & $6(26.1)$ & 0.744 \\
\hline Klebsiella pneumoniae & $8(38.1)$ & $7(30.4)$ & 0.752 \\
\hline Pseudomonas aeruginosa & $1(4.8)$ & $6(26.1)$ & 0.097 \\
\hline Acinetobacter baumannii & $1(4.8)$ & $1(4.3)$ & 1.000 \\
\hline
\end{tabular}

COPD chronic obstructive pulmonary disease, ARDS acute respiratory distress syndrome, ED emergency department, APACHE I/ Acute Physiology and Chronic Health Evaluation II, mSOFA modified Sequential Organ Failure Assessment

In subgroup analysis, there were no clinical and microbiological differences in outcomes in sepsis and septic shock patients who escalated from other wards to ICU. However, patients who admitted from ED had a higher trend of microbiological cure rate in the high-dose group $(66.7 \%$ vs. $41.2 \% ; P$ value $=0.181)$. In patients admitted from ED with mSOFA score $\geq 7$, high-dose meropenem demonstrated significantly better microbiological cure compared with standard-dose $(75 \%(9 / 12)$ vs. $20 \%(2 / 10) ; P$ value $=0.03)$. Likewise, in patients admitted from ED and had APACHE II score $>20$, the high-dose group had a higher microbiological cure rate 
Table 2 Duration of meropenem and concomitant antimicrobial therapy

\begin{tabular}{llll}
\hline & Standard-dose meropenem $(n=38)$ & High-dose meropenem $(n=38)$ & $P$ value \\
\hline Day of meropenem therapy, median (IQR) & $6(3-16)$ & $5(3-21)$ & $0.035^{*}$ \\
Concomitant antimicrobial therapy, $n(\%)$ & $16(42.1)$ & $19(50)$ & 0.646 \\
Colistin, $n(\%)$ & $3(18.8)$ & $2(10.5)$ & $6(31.6)$ \\
Vancomycin, $n(\%)$ & $7(43.8)$ & $4(21.1)$ & 0.642 \\
Cotrimoxazole, $n(\%)$ & $1(6.3)$ & $3(15.8)$ & 0.503 \\
Levofloxacin, $n(\%)$ & $0(0)$ & $3(15.8)$ & 0.347 \\
Amikacin, $n(\%)$ & $4(25)$ & $2(10.5)$ & 0.234 \\
Azithromycin, $n(\%)$ & $1(6.3)$ & $2(10.5)$ & 0.677 \\
Oseltamivir, $n(\%)$ & $2(12.5)$ & $25(65.8)$ & 1.000 \\
De-escalation, $n(\%)$ & $21(55.3)$ & 1.000 \\
\hline
\end{tabular}

*Statistically significant difference

$(83.3 \%(10 / 12)$ vs. $28.6 \%(2 / 7) ; P$ value $=0.045)$ than the standard-dose group. In patients admitted from ED who used the mechanical ventilator, the high-dose group had a higher microbiological cure rate than the standard-dose group (87.5\% (7/8) vs. 23.1\% (3/13); $P$ value $=0.008)($ Fig. 3$)$. Moreover, even though urinary tract infections (UTI) were higher in the high-dose group (23.7\%) than in the standarddose group (5.3\%), $P$ value $=0.047$, they did not affect any significant changes in both primary and secondary outcomes in bivariate analysis.

For adverse events, diarrhea was reported in 12 patients $(31.6 \%)$ in the standard-dose group and 8 patients (21.1\%) in the high-dose group with no statistical significance $(P$ value $=0.435)$. However, there were no other adverse events, such as seizure or Clostridium difficile infection, found in the two groups, as shown in Table 3.

\section{Discussion}

In our study, we found no difference in delta mSOFA between the standard-dose and the high-dose meropenem group. In addition, each component of the SOFA score was not worsening in both of the standard-dose and the high-dose groups. The explanation of this comparable delta mSOFA between the standard-dose and the high-dose meropenem might be as follows. First, the pathophysiology of sepsis and septic shock starts from dysregulated host responses to infection. A combination of hemodynamic and cellular failures were then followed constantly [13]. Appropriate antibiotic therapy at an early point of sepsis diagnosis will help in reducing microbial load. Consequently, the burden from microbial toxin released or produced by bacteria will be terminated [14]. Inflammatory response leading to tissue

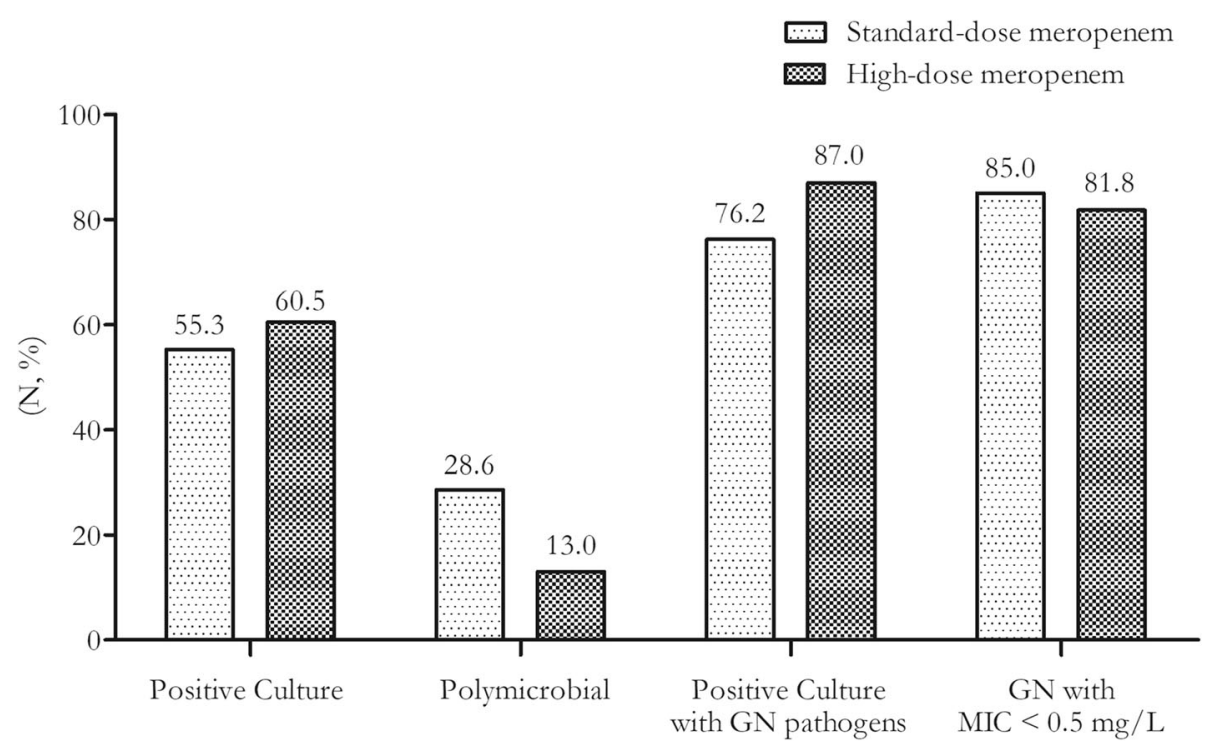

Fig. 2 Bacterial isolates. GN, gram-negative bacteria; MIC, minimum inhibitory concentration 
Table 3 Primary and secondary outcomes

\begin{tabular}{|c|c|c|c|}
\hline Outcomes & Standard-dose meropenem $(n=38)$ & High-dose meropenem $(n=38)$ & $P$ value \\
\hline \multicolumn{4}{|l|}{ Primary outcome } \\
\hline Delta mSOFA score, median (IQR) & -1 ( -6 to 3$)$ & -1 ( -9 to 4$)$ & 0.746 \\
\hline Delta respiratory score & -1 ( -3 to 2$)$ & $-1(-4$ to 2$)$ & 0.136 \\
\hline Delta cardiovascular score & $0(-4$ to 3$)$ & -1 ( -4 to 3$)$ & 0.599 \\
\hline Delta liver score & $0(-1$ to 2$)$ & $0(-1$ to 2$)$ & 0.570 \\
\hline Delta renal score & $0(-1$ to 2$)$ & $0(-2$ to 1$)$ & 0.201 \\
\hline Delta coagulation score & $0(-2$ to 3$)$ & $0(-1$ to 3$)$ & 0.052 \\
\hline \multicolumn{4}{|l|}{ Secondary outcomes } \\
\hline 14-day mortality, $n(\%)$ & $6(15.8)$ & $4(10.5)$ & 0.736 \\
\hline 28-day mortality, $n(\%)$ & $17(44.7)$ & $13(34.2)$ & 0.482 \\
\hline Clinical cure, $n(\%)$ & $33(86.8)$ & $33(86.8)$ & 1.000 \\
\hline Microbiological cure, $n(\%)$ & $17(44.7)$ & $21(55.3)$ & 0.492 \\
\hline Vasopressor-free days, median (IQR) & $24.5(0-27)$ & $25(0-27)$ & 0.350 \\
\hline Ventilator-free days, median (IQR) & $11.5(0-24)$ & $10.0(0-28)$ & 0.819 \\
\hline ICU-free days, median (IQR) & $0.5(0-19)$ & $9.5(0-20)$ & 0.819 \\
\hline Hospital-free days, median (IQR) & $0(0-74)$ & $37.5(0-72)$ & 0.819 \\
\hline \multicolumn{4}{|l|}{ Adverse events } \\
\hline Diarrhea, n (\%) & $12(31.6)$ & $8(21.1)$ & 0.435 \\
\hline
\end{tabular}

mSOFA modified sequential organ failure assessment score, ICU intensive care unit

injury would then be declined [14]. Since critically ill patients have substantially physiologic changes, the suboptimal concentration of antibiotics was reported $[8,15,16]$. Thus, high-dose antibiotics should be taken into consideration to achieve better clinical outcomes [6]. Nevertheless, cellular failure due to the individual dysregulated host responses to pro-inflammatory cytokine and alteration of cellular immune function may also progress and might not have been helped by the higher dose of antibacterial $[13,17]$. Second, $44.7 \%$ of patients included in our study had a negative culture, which might have been from noninfectious causes, viruses, or fungal infection $[18,19]$. In fact, supportive treatments such as fluid resuscitation, vasopressor therapy, and nursing care were appropriate in both of the standard-dose and the high-dose groups.

For the secondary outcomes, 14-day and 28-day mortality, clinical cure, and vasopressor-, ventilator-, ICU-, and hospital-free days, there were no significant differences between the two meropenem dosing strategies. Since the sample size in our study was explicitly calculated focused on delta mSOFA score, the sample size might be insufficiently powered to detect differences of secondary outcomes between two groups. Besides, even though there was a trend of higher ICU- and hospital-free days in the high-dose group, there was no statistical difference between the groups. The secondary outcomes' results might have been confounded by a persistent inflammation-immunosuppression and catabolism syndrome (PICS), and post-sepsis syndrome which are increasingly found in critically ill patients [20, 21]. Therefore, increased risk of death and reduced healthrelated quality of life would be associated with long-term effect consequences reflecting the secondary outcomes [21]. Moreover, serious adverse drug events, seizure, and Clostridium difficile infection were not reported in the high-dose group. Since we excluded patients who might

Table 4 Pharmacodynamic target of the time above the minimum inhibitory concentration

\begin{tabular}{lll}
\hline MIC $(\mathrm{mg} / \mathrm{L})$ & Standard-dose meropenem $(n=8)$ & High-dose meropenem $(n=10)$ \\
\hline Identified MIC, $n$ & 3 & 5 \\
- Achievement of 40\%T>MIC, $n(\%)$ & $3(100 \%)$ & $5(100 \%)$ \\
- Achievement of 100\%T>MIC, $n(\%)$ & $2(66.7 \%)$ & $5(100 \%)$ \\
MIC $=8, n$ & 8 & 10 \\
- Achievement of 40\%T>MIC, $n(\%)$ & $5(62.5 \%)$ & $9(90 \%)$ \\
- Achievement of 100\%T>MIC, $n(\%)$ & $2(25 \%)$ & $4(40 \%)$ \\
\hline
\end{tabular}

MIC minimum inhibitory concentration, $\% T>M I C$ percent of the time of antibiotic concentrations above the minimum inhibitory concentration 


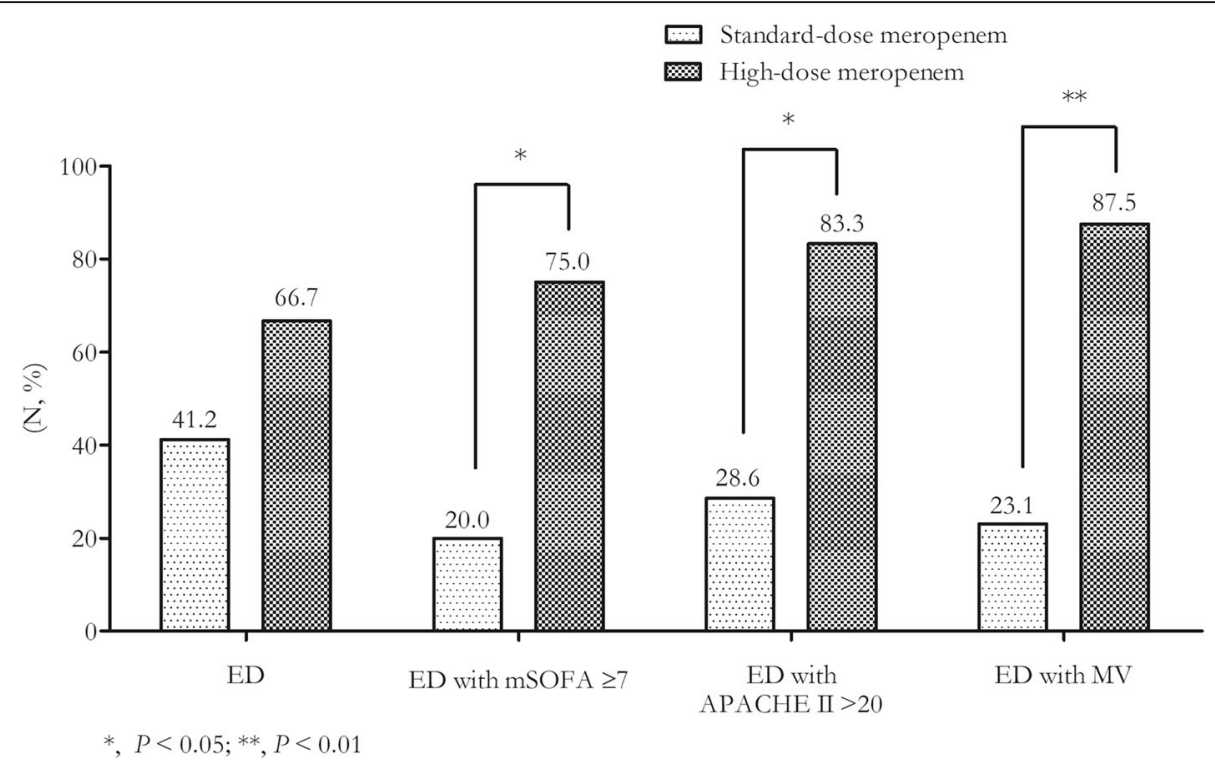

Fig. 3 Comparison of microbiological cure rate in patients who admitted from the emergency department. ED, emergency department; mSOFA, modified sequential organ failure assessment score; APACHE II, Acute Physiology and Chronic Health Evaluation II; MV, mechanical ventilation

possibly have risk for potential toxicity from meropenem accumulation and we adjusted the meropenem dosing regimen by patients' renal functions, no dose-related serious adverse events was found.

Although the duration of prescribed meropenem in the standard-dose group was significantly shorter than the high-dose group ( 5 days vs. 6 days; $P$ value $=0.035$ ), the high-dose group had still shown a trend toward increasing in microbiological cure compared to the standard-dose group $(55.3 \%$ vs. $44.7 \% ; P$ value $=0.492)$. This was because the higher concentration of meropenem at the site of infection was required in order to get a better microbiological cure $[22,23]$. Regarding the meropenem concentration in our study, both regimens of meropenem achieved PD target of $40 \%$ of the time above the identified MIC. Considering suspected resistant bacteria, achieving the PD target of $40 \% \mathrm{~T}>\mathrm{MIC}$ of 8 $\mathrm{mg} / \mathrm{L}$ was higher in the high-dose group (9/10 patients, $90 \%$ ) compared with the standard-dose group (5/8 patients, 62.5\%). Likewise, achieving the PD target of $100 \% \mathrm{~T}>\mathrm{MIC}$ was higher in the high-dose group (4/10 patients, $40 \%)$ compared to the standard-dose group (2/ 8 patients, 25\%). Since there was a small number of blood samples collected, statistical differences were not found. Frippiat et al. performed Monte Carlo simulation to estimate meropenem concentration at epithelial lining fluid (ELF) for meropenem $2 \mathrm{~g}$ every $8 \mathrm{~h}$ and $1 \mathrm{~g}$ every 8 h. At MIC $0.5 \mathrm{mg} / \mathrm{L}$, which was the most cut point MIC in our study, prolonged infusions of meropenem $2 \mathrm{~g}$ every $8 \mathrm{~h}$ demonstrated $\geq 90 \%$ probability of target attainment (PTA) of $100 \% \mathrm{~T}>\mathrm{MIC}$. While meropenem $1 \mathrm{~g}$ every $8 \mathrm{~h}$ demonstrated $\geq 90 \%$ PTA of $40 \% \mathrm{~T}>\mathrm{MIC}$ [24].
Consequently, an increasing meropenem plasma concentration in the high-dose group might assumingly have an increasing trend in meropenem concentration in ELF, which was a target site in almost half of the patients in our study. Nevertheless, we still did not find statistically significant difference between the two groups regarding microbiological cure.

Therefore, we performed subgroup analysis, which showed there were 3 groups of patients that would benefit from the high-dose meropenem. In patients admitted from the ED with mSOFA score $\geq 7$, the high-dose group demonstrated significantly better microbiological cure compared with the standard-dose group $(75 \%(9 / 12$ patients) vs. $20 \%(2 / 10$ patients $) ; P$ value $=0.03)$. Similarly, in patients admitted from the ED with an APACHE II score $>20$ and patients admitted from the ED with mechanical ventilators, the high-dose group had a higher microbiological cure rate than the standard-dose group (83.3\% (10/12 patients) vs. $28.6 \%$ (2/7 patients); $P$ value $=0.045$ and $87.5 \%$ (7/8 patients) vs. $23.10 \%(3 / 13$ patients); $P$ value $=0.008$ ). Since this is the first study identifying different outcomes between two meropenem dosing regimens in ED, high-severity patients might get better outcomes from the higher dose of meropenem. The reason for better microbiological cure might be from higher tissue penetration and overall maintenance of plasma concentration in severe patients. Goncalves et al. found that critically ill patients with a greater baseline severity had a significant increase in the volume of distribution, leading to low meropenem concentrations. Decreased antibiotic concentration at the infection site was eventually found from reduced microvascular 
perfusion in critically ill patients $[25,26]$. However, the high-dose meropenem did not demonstrate better clinical outcomes, delta mSOFA, mortality, vasopressor-free days, ventilator-free days, ICU-free days, and hospitalfree days, in these 3 groups of patients.

However, our study has several limitations. Firstly, since this was the first study comparing $3 \mathrm{~g} /$ day with $6 \mathrm{~g} /$ day of meropenem, the sample size calculation was based on the delta mSOFA from the previous 3 months before patient recruitment, but not from the differences of delta mSOFA between two meropenem dosing. Therefore, the sample size might not have been enough to detect significances between the two dosing strategies. Second, the negative culture found in about $50 \%$ of patients in our study, impact on different meropenem dosing could not be assumed. Early differentiation of nonbacterial infection from bacterial infection before empirical therapy has not yet been easily done in our institution. Third, almost all of our patients' relatives experienced grief when confronted with a deteriorating phase of their love ones. Informed consent regarding blood sample collection was too difficult when there was a treatment limitation decision. For that reason, meropenem blood concentration collection was performed in only $5 \%$ of our recruited patients. Lastly, pathogens identified in this study had relatively low MICs. Narrowing the group of patients that might benefit from the highdose group might be an option. Patient medical history, local antibiogram, and epidemiology of antimicrobial resistance in each area might be needed to modify inclusion criteria in order to specify the group of patients likely to be infected by higher MICs.

\section{Conclusions}

Empirical therapy with a high-dose of meropenem in critically ill patients showed no statistical difference in patient's clinical outcomes compared to a standard-dose of meropenem. However, subgroup analysis showed that patients who were admitted from ED and had mSOFA score $\geq 7$ or APACHE II score $>20$ or had been on mechanical ventilator manifested superior microbiological cure rate in sepsis or septic shock patients admitted from ED.

\begin{abstract}
Abbreviations
mSOFA: Modified sequential organ failure assessment score; ED: Emergency department; PK: Pharmacokinetic; MDR: Multidrug-resistant; ESBL: Extendedspectrum beta-lactamase; PD: Pharmacodynamic; ICU: Intensive care unit; fT >MIC: Time of free antibiotic concentrations above the minimum inhibitory concentration; CICr: Creatinine clearance; APACHE II: Acute Physiology and Chronic Health Evaluation II; LC-MS/MS: Liquid chromatography-tanden mass spectrometry; CDC: Centers for Disease Control and Prevention; SD: Standard deviation; PTA: Probability of target attainment; ELF: Epithelial lining fluid
\end{abstract}

\section{Acknowledgements}

We would like to thank to Panadda Panusitthikorn, Suthinee Taesotikul, Auranee Trisataya, and all of the staff at the medical intensive care unit at Ramathibodi hospital for their help throughout the study. I wish to thank the
Lertwattanachai family and Thanatporn Rungrojvuttikul for their support and encouragement throughout the research period.

\section{Authors' contributions}

TP participated in the study design, data collection and analysis, and drafted the manuscript. PM participated in the study design and revised the manuscript. VT participated in the study design and data collection. PS participated in the data collection. JS and SA participated in the blood sample collection and measured drug concentration. PD participated in the study design, data analysis, and revised the manuscript. All authors read and approved the final manuscript.

\section{Funding}

Tospon Lertwattanachai is a research fellow and currently supported by a residency training program, Faculty of Pharmacy, Mahidol University. Pitchaya Dilokpattanamongkol also partially received funding from the Dr. Kasem Foundation, Bangkok, Thailand. Both the residency training program and Dr. Kasem foundation had not been involved in the study design, data collection, analysis, data interpretation, and manuscript preparation.

\section{Availability of data and materials}

The datasets used and/or analyzed during the current study are available from the corresponding author on reasonable request.

\section{Ethics approval and consent to participate}

The protocol was approved by the committee on Human Rights Related to Research Involving Human Subjects at the Faculty of Medicine Ramathibodi Hospital, Mahidol University (MURA2017/488). An informed consent was required before meropenem was given and was signed by the patient or legal representative.

\section{Consent for publication}

Written informed consent was obtained from the patient or legal representative for publication of their individual details and accompanying images in this manuscript. The consent forms are held by the authors' institution and are available for review by the Editor-in-Chief.

\section{Competing interests}

The authors declare that they have no competing interests.

\section{Author details}

'Department of Pharmacy, Faculty of Pharmacy, Mahidol University, Bangkok 10400, Thailand. 'Department of Critical Care Medicine, Faculty of Medicine, Ramathibodi Hospital, Mahidol University, Bangkok, Thailand. ${ }^{3}$ Piyavate Hospital, Bangkok, Thailand. ${ }^{4}$ Department of Emergency Medicine, Faculty of Medicine, Ramathibodi Hospital, Mahidol University, Bangkok, Thailand.

${ }^{5}$ Toxicology Laboratory, Department of Pathology, Faculty of Medicine, Ramathibodi Hospital, Mahidol University, Bangkok, Thailand.

Received: 18 November 2019 Accepted: 26 March 2020

Published online: 15 April 2020

\section{References}

1. Cecconi M, Evans L, Levy M, Rhodes A. Sepsis and septic shock. Lancet. 2018;392(10141):75-87.

2. Ulldemolins M, Vaquer S, Llauradó-Serra M, Pontes C, Calvo G, Soy D, et al. Beta-lactam dosing in critically ill patients with septic shock and continuous renal replacement therapy. Crit Care. 2014;18(3):227.

3. Roberts JA, Kumar A, Lipman J. Right dose, right now: customized drug dosing in the critically ill. Critical care medicine. 2017:45(2):331-6.

4. Delattre IK, Taccone FS, Jacobs F, Hites M, Dugernier T, Spapen H, et al. Optimizing $\beta$-lactams treatment in critically-ill patients using pharmacokinetics/pharmacodynamics targets: are first conventional doses effective? Expert review of anti-infective therapy. 2017;15(7):677-88.

5. Blot SI, Pea F, Lipman J. The effect of pathophysiology on pharmacokinetics in the critically ill patient-concepts appraised by the example of antimicrobial agents. Advanced drug delivery reviews. 2014;77:3-11

6. Masich AM, Heavner MS, Gonzales JP, Claeys KC. Pharmacokinetic/ pharmacodynamic considerations of beta-lactam antibiotics in adult critically ill patients. Current infectious disease reports. 2018;20(5):9. 
7. Suwantarat N, Carroll KC. Epidemiology and molecular characterization of multidrug-resistant Gram-negative bacteria in Southeast Asia. Antimicrobial Resistance Infection Control. 2016;5(1):15.

8. Roberts JA, Paul SK, Akova M, Bassetti M, De Waele JJ, Dimopoulos G, et al. DALl: defining antibiotic levels in intensive care unit patients: are current $\beta$ lactam antibiotic doses sufficient for critically ill patients? Clinical infectious diseases. 2014;58(8):1072-83.

9. Rizk NA, Kanafani ZA, Tabaja HZ, Kanj SS. Extended infusion of beta-lactam antibiotics: optimizing therapy in critically-ill patients in the era of antimicrobial resistance. Expert review of anti-infective therapy. 2017;15(7): 645-52.

10. Burger R, Guidi M, Calpini V, Lamoth F, Decosterd L, Robatel C, et al. Effect of renal clearance and continuous renal replacement therapy on appropriateness of recommended meropenem dosing regimens in critically ill patients with susceptible life-threatening infections. Journal of Antimicrobial Chemotherapy. 2018;73(12):3413-22.

11. Sjövall F, Alobaid AS, Wallis SC, Perner A, Lipman J, Roberts JA. Maximally effective dosing regimens of meropenem in patients with septic shock. J Antimicrobial Chemother. 2017;73(1):191-8.

12. Jaruratanasirikul $\mathrm{S}$, Thengyai $\mathrm{S}$, Wongpoowarak W, Wattanavijitkul T, Tangkitwanitjaroen $\mathrm{K}$, Sukarnjanaset W, et al. Population pharmacokinetics and Monte Carlo dosing simulations of meropenem during the early phase of severe sepsis and septic shock in critically ill patients in intensive care units. Antimicrobial agents and chemotherapy. 2015;59(6):2995-3001.

13. Lelubre C, Vincent J-L. Mechanisms and treatment of organ failure in sepsis. Nature Reviews Nephrology. 2018;14(7):417-27.

14. Kumar A. An alternate pathophysiologic paradigm of sepsis and septic shock: implications for optimizing antimicrobial therapy. Virulence. 2014;5(1): 80-97.

15. Ehmann L, Zoller M, Minichmayr IK, Scharf C, Maier B, Schmitt MV, et al. Role of renal function in risk assessment of target non-attainment after standard dosing of meropenem in critically ill patients: a prospective observational study. Crit Care. 2017;21(1):263.

16. Wu C-C, Tai C-H, Liao W-Y, Wang C-C, Kuo C-H, Lin S-W, et al. Augmented renal clearance is associated with inadequate antibiotic pharmacokinetic/ pharmacodynamic target in Asian ICU population: a prospective observational study. Infection Drug Resistance. 2019;12:2531.

17. Delano MJ, Ward PA. The immune system's role in sepsis progression, resolution, and long-term outcome. Immunological reviews. 2016;274(1): 330-53.

18. de Prost N, Razazi K, Brun-Buisson C. Unrevealing culture-negative severe sepsis. Crit Care. 2013;17(5):1001.

19. Vincent J-L, Sakr Y, Sprung CL, Ranieri VM, Reinhart K, Gerlach H, et al. Sepsis in European intensive care units: results of the SOAP study. Critical care medicine. 2006;34(2):344-53.

20. Mira JC, Gentile LF, Mathias BJ, Efron PA, Brakenridge SC, Mohr AM, et al. Sepsis pathophysiology, chronic critical illness and PICS. Crit Care Med. 2017:45(2):253

21. Mostel Z, Perl A, Marck M, Mehdi SF, Lowell B, Bathija S, et al. Post-sepsis syndrome-an evolving entity that afflicts survivors of sepsis. Mol Med. 2020; 26(1):6.

22. Yu Z, Pang X, Wu X, Shan C, Jiang S. Clinical outcomes of prolonged infusion (extended infusion or continuous infusion) versus intermittent bolus of meropenem in severe infection: A meta-analysis. PloS one. 2018; 13(7):e0201667.

23. Liang SY, Kumar A. Empiric antimicrobial therapy in severe sepsis and septic shock: optimizing pathogen clearance. Curr Infectious Dis Rep. 2015;17(7):36.

24. Frippiat F, Musuamba FT, Seidel L, Albert A, Denooz R, Charlier C, et al. Modelled target attainment after meropenem infusion in patients with severe nosocomial pneumonia: the PROMESSE study. The Journal of antimicrobial chemotherapy. 2015;70(1):207-16.

25. Goncalves-Pereira J, Silva NE, Mateus A, Pinho C, Povoa P. Assessment of pharmacokinetic changes of meropenem during therapy in septic critically ill patients. BMC Pharmacol Toxicol. 2014;15(1):21.

26. Gonçalves-Pereira J, Póvoa P. Antibiotics in critically ill patients: a systematic review of the pharmacokinetics of $\beta$-lactams. Crit Care. 2011;15(5):R206.

\section{Publisher's Note}

Springer Nature remains neutral with regard to jurisdictional claims in published maps and institutional affiliations.

Ready to submit your research? Choose BMC and benefit from:

- fast, convenient online submission

- thorough peer review by experienced researchers in your field

- rapid publication on acceptance

- support for research data, including large and complex data types

- gold Open Access which fosters wider collaboration and increased citations

- maximum visibility for your research: over $100 \mathrm{M}$ website views per year

At BMC, research is always in progress.

Learn more biomedcentral.com/submissions 